\title{
Gabapentin enacarbil in subjects with moderate to severe primary restless legs syndrome with and without severe sleep disturbance: an integrated analysis of subjective and novel sleep endpoints from two studies
}

This article was published in the following Dove Press journal:

Journal of Parkinsonism and Restless Legs Syndrome

16 May 2013

Number of times this article has been viewed

\author{
Richard K Bogan' \\ Aaron Ellenbogen ${ }^{2}$ \\ Philip M Becker ${ }^{3}$ \\ Clete Kushida ${ }^{4}$ \\ Eric Ball ${ }^{5}$ \\ William G Ondo ${ }^{6}$ \\ Christine K Caivano ${ }^{7}$ \\ Sarah Kavanagh ${ }^{7}$ \\ 'SleepMed, Columbia, SC, ${ }^{2}$ Quest \\ Research Institute, Farmington Hills, \\ MI, ${ }^{3}$ Sleep Medicine Associates of \\ Texas, Dallas, TX, ${ }^{4}$ Division of Sleep \\ Medicine, Department of Psychiatry \\ and Behavioral Sciences, Stanford \\ Center for Human Sleep Research, \\ Stanford, CA, ${ }^{5}$ Walla Walla Clinic, \\ Walla Walla, WA, 'University of \\ Texas Health Science Center. \\ Houston, TX, ${ }^{7}$ Global Regulatory \\ Affairs (CKC)* and Neurosciences \\ MDC (SK), GlaxoSmithKline, \\ Research Triangle Park, NC, USA \\ *Development Sciences department \\ at the time of the analysis
}

\begin{abstract}
Purpose: The aim of the study reported here was assessment of subjective and novel sleep endpoints, according to sleep disturbance severity at baseline, in adult subjects with moderate to severe primary restless legs syndrome (RLS) treated with gabapentin enacarbil (GEn) $1200 \mathrm{mg}$ or placebo.
\end{abstract}

Methods: Integrated analysis of two 12-week randomized trials in subjects with RLS was undertaken. Sleep outcomes from the Medical Outcomes Study (MOS) Sleep Scale and the Post Sleep Questionnaire were evaluated. Novel sleep endpoints derived from the 24-Hour RLS Symptom Diary were compared with similar endpoints derived from the Pittsburgh Sleep Diary (PghSD). Subjects were divided into two subgroups based on their level of sleep disturbance (responses to item 4 of the International Restless Legs Scale) at baseline. Data were analyzed using a last observation carried forward approach.

Results: The modified intent-to-treat population comprised 427 subjects (GEn $1200 \mathrm{mg}$, $\mathrm{n}=223$; placebo, $\mathrm{n}=204)$. GEn significantly improved all MOS Sleep Scale domain scores from baseline compared with placebo $(P<0.05)$ in both subgroups. Compared with placebo, GEn-treated subjects with very severe to severe sleep disturbance reported higher overall sleep quality, fewer nighttime awakenings, and fewer hours awake per night due to RLS symptoms at Week 12 on the Post Sleep Questionnaire (all $P<0.001$, distribution of responses); sleep quality was the only significant item in those with moderate to no sleep disturbance $(P<0.0001)$. Evaluation of sleep endpoints derived from the 24-Hour RLS Symptom Diary and PghSD yielded similar results.

Conclusion: Once-daily GEn $1200 \mathrm{mg}$ significantly improves subjective sleep outcomes compared with placebo in subjects with RLS, regardless of the severity of sleep disturbance at baseline, although a greater improvement in sleep assessments may be observed in subjects with very severe to severe sleep disturbance than in those with moderate to no disturbance. Similar patterns were observed between treatment groups when comparing sleep endpoints derived from the PghSD and the novel sleep endpoints derived from the 24-Hour RLS Symptom Diary.

Keywords: 24-Hour RLS Symptom Diary, Pittsburgh Sleep Diary, Medical Outcomes Study Sleep Scale, Post Sleep Questionnaire

\section{Introduction}

Restless legs syndrome (RLS) is a common neurologic disorder characterized by an urge to move the legs, often accompanied by unpleasant or painful leg sensations 
that are partially or totally relieved by movement. ${ }^{1}$ Owing to the occurrence or worsening of RLS in the evening or night, more than $75 \%$ of subjects report at least one sleep-related impairment. ${ }^{2}$ RLS symptoms may delay sleep onset, decrease total sleep time, and cause multiple awakenings, resulting in significant sleep disturbance. ${ }^{3,4}$ This in turn may lead to a subsequent increase in daytime fatigue and sleepiness. ${ }^{2,5}$ Comparative studies have shown that subjects with RLS suffer reduced sleep compared with both healthy controls ${ }^{6}$ and those with major depressive disorders. ${ }^{7}$ Further, poor sleep patterns at RLS diagnosis appear to be predictive of poor psychological functioning about 3.5 years later. ${ }^{8}$

Gabapentin enacarbil (GEn) is an actively transported prodrug of gabapentin that is indicated for the treatment of moderate to severe primary RLS in adults. ${ }^{9}$ It is absorbed throughout the large and small intestine by high-capacity nutrient transporters and is rapidly and extensively hydrolyzed to gabapentin, providing sustained, dose-proportional gabapentin exposure. ${ }^{10-12}$ GEn conversion to gabapentin following absorption is efficient and provides more predictable gabapentin exposure and increased bioavailability than milligram-equivalent doses of oral gabapentin. ${ }^{12}$ As GEn is a prodrug of gabapentin, its therapeutic effects in RLS are attributable to gabapentin; however, the precise mechanism by which gabapentin treats RLS is unknown. ${ }^{9}$

The efficacy and tolerability of GEn in adults with moderate to severe primary RLS have been demonstrated in two Phase III, 12-week, multicenter, placebo-controlled, randomized clinical trials. ${ }^{13,14}$ These showed that, compared with placebo, treatment with GEn $600 \mathrm{mg}$ (Study XP053) (4 $^{14}$ and $1200 \mathrm{mg}$ (Studies XP052 and XP053) ${ }^{13,14}$ is associated with significant improvements in both restlessness and sleep disturbance, as measured using established tools such as the International Restless Legs Scale (IRLS), which measures specific RLS-related symptoms and symptom impact; the Clinical Global Impression - Improvement (CGI-I) scale, which assesses global change in condition; the Medical Outcomes Study (MOS) Sleep Scale, which provides a general measure of several sleep domains; the Post Sleep Questionnaire (PSQ), which evaluates sleep disturbance in RLS; and the Pittsburgh Sleep Diary (PghSD), which quantifies sleep and waking behaviors. GEn $600 \mathrm{mg}$ is the daily dose approved by the US Food and Drug Administration for the treatment of moderate to severe primary RLS in adults.

In addition to such established tools, both studies also used a novel instrument to monitor RLS symptoms: the 24-Hour RLS Symptom Diary. This patient diary, developed by XenoPort (Santa Clara, CA, USA) for use in clinical trials, assesses whether a subject experiences RLS symptoms over a 24-hour period. Patients have to indicate whether symptoms are present and, if so, their severity, and also record when they are asleep and symptoms cannot be measured. In both Studies XP052 and XP053, GEn-treated groups showed a delayed time of onset of RLS symptoms within the 24-hour period compared with placebo groups, as well as a higher percentage of symptom-free subjects. ${ }^{13,14}$

Here, we present a retrospective integrated analysis of Studies XP052 and XP053, assessing subjective sleep outcomes and the tolerability of once-daily GEn $1200 \mathrm{mg}$ using pooled data from the two trials. The objective of such analysis was two fold: first to evaluate - using data pooled according to severity of sleep disturbance at study entry whether baseline sleep disturbance could have an effect on sleep outcomes, as the interaction between treatment effect and baseline sleep disturbance was not assessed in the original studies; second, to assess how informative two novel sleep endpoints derived from the 24-Hour RLS Symptom Diary were. These were analyzed alongside well-established subjective sleep outcomes from the MOS Sleep Scale and the PSQ for exploration and concurrence, and then compared with similar endpoints derived from the PghSD.

\section{Subjects and methods Study design}

Data from two 12-week, randomized, double-blind, parallelgroup, placebo-controlled Phase III trials of GEn versus placebo were integrated. In Study XP052 (XenoPort protocol number; clinical trials.gov identifier NCT00298623 ${ }^{15}$ ), which was conducted at 22 centers in the USA, subjects were randomized to receive GEn $1200 \mathrm{mg}$ or placebo. ${ }^{13}$ In Study XP053 (XenoPort protocol number; clinical trials. gov identifier NCT00365352), ${ }^{16}$ conducted at 28 centers in the USA, subjects were randomized to receive GEn $600 \mathrm{mg}$, $1200 \mathrm{mg}$, or placebo. ${ }^{14}$ In both studies, GEn or placebo was administered once daily at 5 pm with food; GEn was initiated at a dose of $600 \mathrm{mg}$ with subsequent titration to $1200 \mathrm{mg}$ after 3 days. At the end of the study or at early termination (ET), subjects receiving GEn $1200 \mathrm{mg}$ had their dose tapered over 7 days.

\section{Subjects}

Key inclusion and exclusion criteria were similar for each study and have been described previously. ${ }^{13,14}$ Briefly, the studies included adults with moderate to severe primary RLS as per International Restless Legs Syndrome Study Group diagnostic criteria, with RLS symptoms for $\geq 15$ days during 
the month prior to screening and for $\geq 4$ nights during the 7-day baseline period, and with a total IRLS score of $\geq 15$ at baseline. Exclusion criteria included secondary RLS, a history of RLS symptom augmentation, or rebound with previous dopamine agonist treatment, and neurologic, sleep, or movement disorders other than RLS.

For this analysis, subjects were divided into two sleep disturbance subgroups (very severe to severe sleep disturbance or moderate to no sleep disturbance) based on their response to IRLS item 4 at baseline. ${ }^{17}$ Subjects were asked, "Overall, how severe is your sleep disturbance from your RLS symptoms?" and were allocated to one of the two subgroups according to the following responses: "very severe" and "severe" versus "moderate," "mild," or "none."

All subjects provided institutional review board-approved written informed consent prior to study participation. The study was conducted in accordance with Good Clinical Practice and the guiding principles of the Declaration of Helsinki.

\section{Study assessments}

\section{Subjective sleep endpoints}

Subjective sleep endpoints from the MOS Sleep Scale, the PSQ, and the PghSD were assessed by baseline sleep disturbance subgroup and by treatment group. For the MOS Sleep Scale, change from baseline in the four domains of sleep disturbance, sleep adequacy, sleep quality, and daytime somnolence was evaluated at Weeks 4, 8, and 12 . For the PSQ, responses to item 1, "overall quality of sleep in the last week"; item 4, "number of awakenings during the night in the past week due to RLS symptoms"; and item 5, "number of hours awake per night in the past week due to RLS symptoms," were assessed at Week 12. Regarding the PghSD, two endpoints were considered and assessed at Weeks 2, 4, 8, and 12: wake time after onset (WASO) and total sleep time (TST). WASO was expressed in minutes and calculated as the mean of available "time awake during the night" values recorded over the 7 days prior to each visit. TST was expressed in hours and calculated using the following formula: (wake-up time - lights out time) - time (minutes) to fall asleep - time awake during the night (minutes); it was analyzed for each of the 7 days prior to each visit and then averaged over that period.

\section{Novel sleep endpoints}

On the 24-Hour RLS Symptom Diary, participants indicated whether symptoms were "not present," "mild," "moderate," or "severe" at 30-minute intervals while awake, and had to record when they were asleep and symptoms could not be measured. The 24-Hour RLS Symptom Diary was completed the day before visits at Weeks 2 and 12/ET.

Two novel sleep endpoints were derived using the 24-Hour RLS Symptom Diary: sleep time (ST) and time awake during the night (TAN). ST was expressed in hours and calculated using the formula: (wake-up time - time asleep) - TAN. In this formula, "time asleep" is the first (earliest) time point occurring after $5.59 \mathrm{pm}$ when the subject indicated they were asleep; "wake-up time" is the first (earliest) time point occurring after "time asleep" when the subject indicated they were awake and stayed awake at all subsequent time points; and "TAN" is the number of intervals occurring after initial "time asleep" during the night and before "wake-up time" in which the subject indicated they were awake, multiplied by 30 minutes.

\section{Sleep-related tolerability endpoints}

Somnolence and sedation, reported as treatment-emergent adverse events (AEs), were summarized (as number of patients [\%]) for both subgroups, including those leading to withdrawal from the studies. The change from baseline in Epworth Sleepiness Scale (ESS) total score was assessed for the overall group of subjects and for each subgroup.

\section{Statistical analyses}

Changes from baseline for the MOS Sleep Scale, ST, TST, and WASO were analyzed using a parametric analysis of covariance (ANCOVA) model adjusted for baseline value, study, pooled site, and treatment. Changes from baseline in TAN were analyzed using non-parametric methods (rank ANCOVA model), adjusted for baseline TAN, pooled site, and study, as the assumption of normality was not met for these data. Categorical PSQ responses were analyzed using a CochranHaenszel mean score test with interval scoring, stratified by pooled site. Clinical Global Impression of Improvement responders were analyzed using a logistic regression model adjusted for pooled site, study, and treatment.

All statistical tests were considered exploratory. For the PSQ, all $P$ values presented are for distribution of responses. No multiplicity adjustments were made for treatment group comparisons, and missing values were imputed using the last observation carried forward (LOCF) technique for the MOS Sleep Scale, PSQ, and PghSD endpoints. The analysis of ST and TAN from the 24-Hour RLS Symptom Diary used observed case data, as no imputation was applied between visits, which was consistent with the original data-handling in the individual studies. If sleep status was missing for a 
particular 30-minute interval within a visit, it was assumed that the subject was awake during that interval.

The safety population comprised all subjects who were enrolled into either study and received at least one dose (or part of a dose) of study medication. The modified intent-totreat population included all subjects in the safety population who completed an IRLS score assessment at baseline and at least one IRLS rating during the treatment period.

\section{Results}

\section{Study population}

The modified intent-to-treat population comprised 427 subjects, 223 of whom received GEn $1200 \mathrm{mg}$ and 204 of whom received placebo. At baseline, 187 (44\%) subjects reported very severe to severe sleep disturbance, and $240(56 \%)$ reported moderate to no sleep disturbance (Table 1). The number of subjects who completed the studies was similar in each subgroup: $83 \%$ in the very severe to severe subgroup and $88 \%$ in the moderate to no sleep disturbance group. Subject demographics were similar across treatment groups and between sleep disturbance subgroups. Baseline characteristics were similar across treatment groups, but baseline IRLS total score and sleep outcomes indicated a greater severity of symptoms in subjects with very severe to severe sleep disturbance (Table 1).

\section{Efficacy}

\section{Subjective sleep endpoints}

GEn 1200 mg significantly improved all MOS Sleep Scale domain scores from baseline to Week 12 LOCF compared with placebo in both subgroups (Figure 1).

On the PSQ, compared with placebo-treated subjects with very severe to severe sleep disturbance, GEn 1200 mg-treated subjects with very severe to severe sleep disturbance reported significantly better overall sleep quality (GEn vs placebo: excellent, $32 \%$ vs $11 \%$; reasonable, $47 \%$ vs $51 \%$; poor, $21 \%$ vs $38 \% ; P=0.0004$ for distribution of responses), fewer nighttime awakenings (GEn vs placebo: none, $47 \%$ vs $21 \%$; $1-2,42 \%$ vs $55 \%$; $3-4,8 \%$ vs $19 \%$; $\geq 5$ times, $2 \%$ vs $5 \%$; $P<0.0001$ for distribution of responses), and fewer hours awake per night due to RLS symptoms (GEn vs placebo: none, $47 \%$ vs $21 \%$; $<1$ hour, $35 \%$ vs $46 \%$; 1 to $<2$ hours, $8 \%$ vs $15 \% ; 2$ to $<3$ hours, $5 \%$ vs $7 \%$; $\geq 3$ hours, $4 \%$ vs $12 \%$; $P=0.0004$ for distribution of responses) at Week 12 LOCF (Figure 2). Subjects with moderate to no sleep disturbance also reported significantly higher overall sleep quality compared with those on placebo (GEn $1200 \mathrm{mg}$ vs placebo: excellent, $23 \%$ vs $9 \%$; reasonable, $70 \%$ vs $69 \%$; poor, $8 \%$ vs $22 \%$; $P<0.0001$ for distribution of responses) at Week 12 LOCF, but no significant difference in nighttime awakenings (GEn

Table I Demographic and baseline characteristics (modified intent-to-treat population)

\begin{tabular}{|c|c|c|c|c|}
\hline & \multicolumn{2}{|l|}{ Placebo $(n=204)$} & \multicolumn{2}{|c|}{ GEn I $200 \mathrm{mg}(\mathrm{n}=223)$} \\
\hline & $\begin{array}{l}\text { Moderate to no } \\
\text { sleep disturbance } \\
(n=I I 2)\end{array}$ & $\begin{array}{l}\text { Very severe to severe } \\
\text { sleep disturbance } \\
(n=92)\end{array}$ & $\begin{array}{l}\text { Moderate to no } \\
\text { sleep disturbance } \\
(\mathrm{n}=\mathrm{I} 28)\end{array}$ & $\begin{array}{l}\text { Very severe to severe } \\
\text { sleep disturbance } \\
(n=95)\end{array}$ \\
\hline Age, years & $49.0(12.53)$ & $50.4(12.48)$ & $49.1(13.31)$ & $53.0(11.77)$ \\
\hline Women, n (\%) & $66(59.00)$ & $56(61.00)$ & $68(53.00)$ & $63(66.00)$ \\
\hline Treated previously for RLS, n (\%) & $30(27.00)$ & $47(51.00)$ & $32(25.00)$ & $40(42.00)$ \\
\hline Duration of RLS symptoms, years & $15.6(14.05)$ & I3.I (II.15) & $12.2(12.12)$ & $16.2(15.06)^{\mathrm{a}}$ \\
\hline 7-day RLS record, days with RLS & $6.1(1.00)$ & $6.2(1.01)$ & $6.0(1.07)$ & $6.2(1.05)$ \\
\hline IRLS total score & $20.5(3.44)$ & $26.4(4.18)$ & $20.0(3.14)$ & $27.4(3.95)$ \\
\hline \multicolumn{5}{|l|}{ Sleep disturbance at baseline } \\
\hline \multicolumn{5}{|l|}{ Baseline MOS sleep scale domain ${ }^{c}$} \\
\hline Sleep disturbance $(0-100)$ & $43.4(19.36)$ & $60.8(19.75)$ & $42.4(19.39)^{d}$ & $63.1(20.96)$ \\
\hline Sleep adequacy $(0-100)$ & $39.2(23.64)$ & $26.5(21.30)$ & $42.0(23.06)^{d}$ & $22.7(19.65)$ \\
\hline Daytime somnolence $(0-100)$ & $30.4(18.31)$ & $41.0(21.15)$ & $33.1(17.85)^{d}$ & $42.7(22.16)$ \\
\hline Sleep quantity (hours) & $6.4(1.00)$ & $5.5(\mathrm{I} .3 \mathrm{I})$ & $6.4(1.26)^{\mathrm{d}}$ & $5.5(1.36)$ \\
\hline \multicolumn{5}{|l|}{ 24-Hour RLS symptom diary } \\
\hline TAN, minutes & $65.8(105.79)^{\mathrm{e}}$ & I0I.4 (I24.28) & $69.4(107.88)^{f}$ & $111.2(153.21)$ \\
\hline ST, hours & $5.7(2.12)^{\mathrm{e}}$ & $5.2(2.20)$ & $5.9(2.05)^{f}$ & $4.5(2.35)$ \\
\hline \multicolumn{5}{|l|}{ PghSD } \\
\hline WASO, minutes & $24.9(26.80)$ & $45.5(42.12)$ & $23.7(21.57)$ & $39.0(34.29)$ \\
\hline TST, hours & $6.8(1.10)$ & $6.5(1.40)$ & $6.8(1.24)$ & 6.1 (I.50) \\
\hline
\end{tabular}

Notes: All values are means (standard deviations) unless stated otherwise. ${ }^{a} \mathrm{n}=94$; ${ }^{\text {nnumber }}$ of days of RLS symptoms experienced during the week prior to baseline; 'comparator mean MOS domain scores based on a healthy cohort sample of US adults were: sleep disturbance $=24.5$, sleep adequacy $=60.5$, daytime somnolence $=21.9$, sleep quantity $=6.8$ hours $^{20} ;{ }^{d} n=127 ;{ }^{e} n=109 ;{ }^{f}=127$.

Abbreviations: GEn, gabapentin enacarbil; IRLS, International Restless Legs Scale; MOS, Medical Outcomes Study; PghSD, Pittsburgh Sleep Diary; RLS, restless legs syndrome; ST, sleep time; TAN, time awake during the night; TST, total sleep time; WASO, wake time after sleep onset. 
A

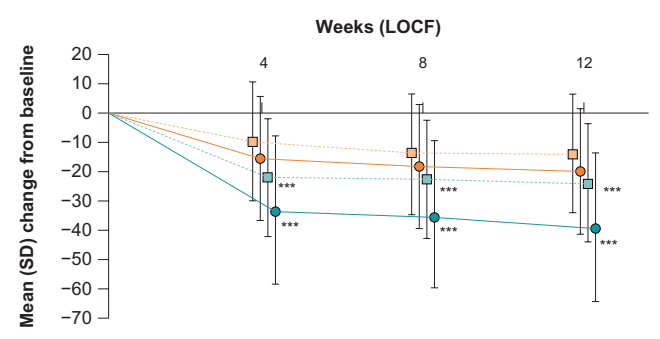

C

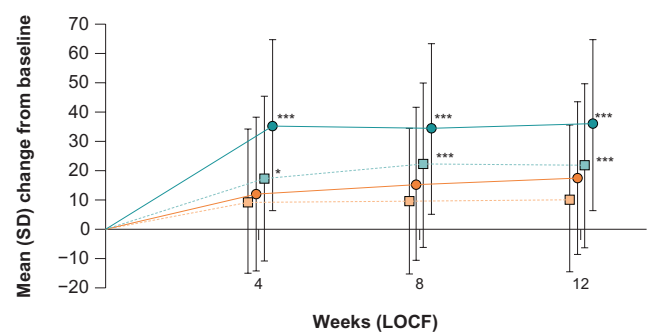

- Very severe to severe sleep disturbance: PBO $(n=92)$

- Very severe to severe sleep disturbance: GEn $1200 \mathrm{mg}(\mathrm{n}=95)$

‥ Moderate to no sleep disturbance: PBO $(n=112)$

․- Moderate to no sleep disturbance: GEn $1200 \mathrm{mg}(n=127)$

B

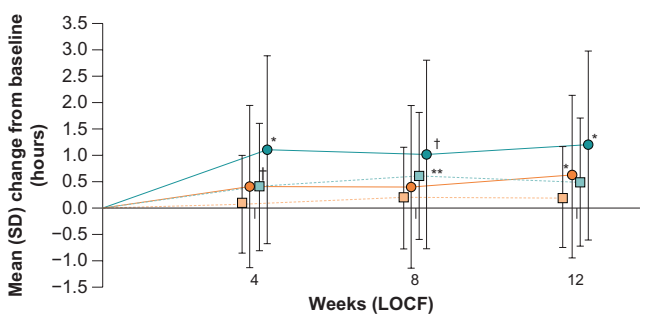

D

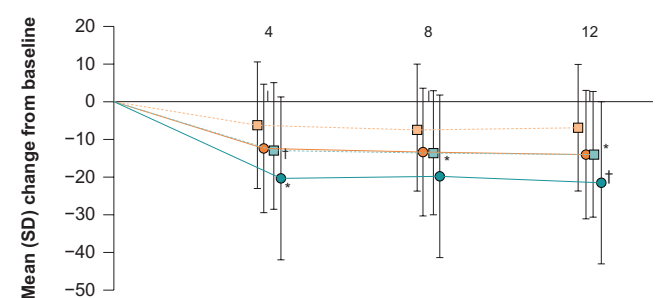

Figure I Mean (SD) change from baseline in domains of the Medical Outcomes Study Sleep Scale by visit (modified intent-to-treat population): (A) sleep disturbance, (B) sleep quantity, (C) sleep adequacy, and (D) daytime somnolence.

Notes: ${ }^{\dagger} P<0.05$; $* P<0.01$; ${ }^{*} * P<0.001$; $* * * P<0.0001$.

Abbreviations: GEn, gabapentin enacarbil; LOCF, last observation carried forward; PBO, placebo; SD, standard deviation.

$1200 \mathrm{mg}$ vs placebo: none, $58 \%$ vs $48 \% ; 1-2,36 \%$ vs $42 \%$; $3-4,6 \%$ vs $7 \%$; $\geq 5$ times, $0 \%$ vs $3 \% ; P=0.0694$ for distribution of responses), or hours awake per night due to RLS symptoms (GEn $1200 \mathrm{mg}$ vs placebo: none, 58\% vs 48\%; $<1$ hour, $31 \%$ vs $38 \%$; 1 to $<2,7 \%$ vs $9 \% ; 2$ to $<3,2 \%$ vs $3 \%$; $\geq 3,2 \%$ vs $2 \% ; P=0.2719$ for distribution of responses) were observed.

\section{Novel sleep endpoints}

On the 24-Hour RLS Symptom Diary, GEn $1200 \mathrm{mg}$ decreased TAN compared with placebo at Week 12/ET in the very severe to severe sleep disturbance subgroup (Figure 3A). The unadjusted mean (standard deviation [SD]) change from baseline was -17.2 (175.36) minutes for placebo and -83.1 (177.11) minutes for GEn $1200 \mathrm{mg}$; the adjusted mean treatment difference (AMTD) was -58.4. As the normality assumptions were not met in this case, the Chisquared value for AMTD using non-parametric ANCOVA was calculated to be $10.9(P=0.0010)$. No significant treatment difference was observed for TAN at Week 12/ET in the moderate to no sleep disturbance subgroup. GEn $1200 \mathrm{mg}$ increased ST compared with placebo at Week 12 in both subgroups (Figure 3B), but no significant treatment difference was observed.

\section{PghSD sleep endpoints}

GEn $1200 \mathrm{mg}$ decreased WASO compared with placebo at Week 12 in both subgroups (Figure 4A). For the very severe to severe sleep disturbance subgroup, the AMTD was -12.7 minutes (95\% confidence interval [CI]: $-20.35,-5.15 ; P=0.0011)$ and for the moderate to no sleep disturbance subgroup it was -4.8 minutes $(95 \%$ CI: $-8.36,-1.15 ; P=0.0100)$. GEn $1200 \mathrm{mg}$ increased TST compared with placebo at Week 12 in both subgroups (Figure 4B), but no significant treatment difference was observed.

\section{Comparison of novel sleep endpoints and the PghSD} Subjects with very severe to severe sleep disturbance at baseline reported less ST and longer TAN compared with subjects with moderate to no sleep disturbance on the 24-Hour RLS Symptom Diary. Likewise, subjects with very severe to severe sleep disturbance at baseline reported less TST and greater WASO compared with subjects with moderate to no sleep disturbance on the PghSD (Table 1). However, there were differences in actual values recorded by the two assessment tools. At baseline, mean TST values taken from the PghSD were between 0.9 and 1.6 hours longer than the ST calculated using the 24-Hour RLS Symptom Diary (Table 1). 

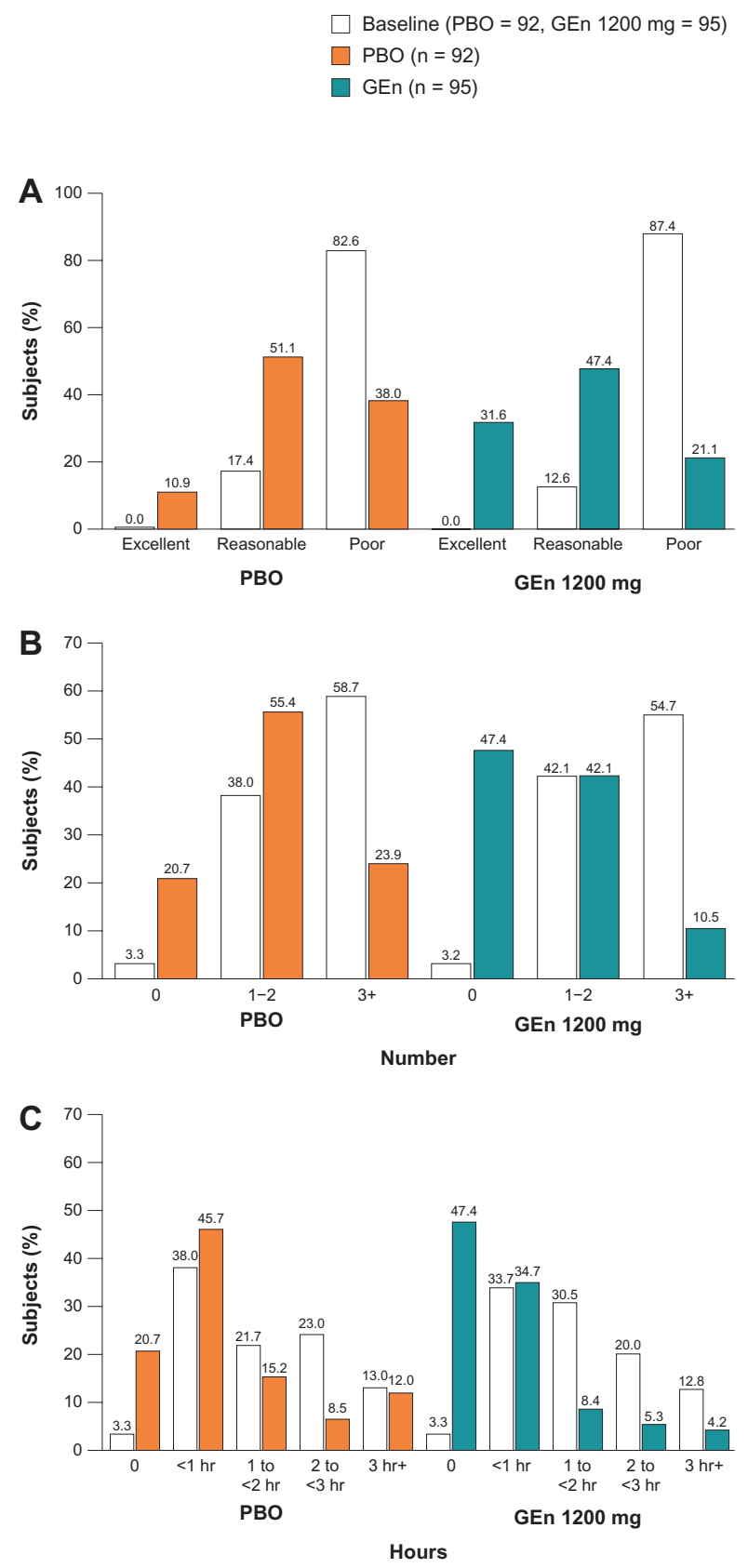

Figure 2 Responses on the Post Sleep Questionnaire items I, 4, and 5 at baseline and Week 12, last observation carried forward for subjects with very severe to severe sleep disturbance at baseline (modified intent-to-treat population): (A) overall quality of sleep in the past week, (B) number of awakenings per night in the past week due to RLS symptoms, and (C) number of hours awake per night in the past week due to RLS symptoms.

Notes: A: $P=0.0004$ for distribution of response. $B: P<0.000$ I for distribution of response. $C: P=0.0004$ for distribution of response.

Abbreviations: GEn, gabapentin enacarbil; PBO, placebo; RLS, restless legs syndrome.

The change from baseline in PghSD TST values were between 0.0 and 0.6 hours shorter than the change from baseline in ST as calculated from the 24-Hour RLS Symptom Diary for GEn subjects with moderate to no and very severe to severe sleep disturbance, respectively (Figure 5A).
Similarly, at baseline, mean WASO values taken from the PghSD were between 41 and 72 minutes shorter than TAN calculated using the 24-Hour RLS Symptom Diary (Table 1). Baseline mean WASO for all subjects was 34.2 minutes for placebo and 30.2 minutes for GEn $1200 \mathrm{mg}$. The change from baseline in WASO values was between 1 and 57 minutes shorter than the change from baseline in TAN, as calculated from the 24-Hour RLS Symptom Diary (Figure 5B).

\section{Safety and tolerability}

The proportion of subjects experiencing AEs of somnolence and/or sedation was similar between the subgroups (very severe to severe sleep disturbance: GEn, 25\% and placebo, $7 \%$; moderate to no sleep disturbance: GEn, 29\% and placebo, $5 \%$ ). Of these, the majority of GEn-treated subjects reported only one occurrence of these events, both in the very severe to severe $(83 \%)$ and in the moderate to no sleep disturbance subgroups (89\%). Most AEs were rated as mild or moderate in intensity. The mean (SD) maximum duration of the somnolence/sedation event in the GEn $1200 \mathrm{mg}$ group was 17.5 (19.22) days for subjects with very severe to severe sleep disturbance and 14.3 (12.34) days for those with moderate to no sleep disturbance. The mean (SD) time to first occurrence of somnolence/sedation AEs was 7.8 (15.46) days in subjects with very severe to severe sleep disturbance and 9.2 (16.84) days for those with moderate to no sleep disturbance.

Baseline mean ESS total score was similar between subgroups: 9.6 for subjects with very severe to severe sleep disturbance (both in the GEn $1200 \mathrm{mg}$ and in the placebo group) and 9.1-9.2 for subjects with moderate to no sleep disturbance. For subjects with very severe to severe sleep disturbance at baseline, there was a treatment benefit for GEn $1200 \mathrm{mg}$ relative to placebo for the change in ESS total score at Week 12/ET (AMTD: $-1.3 ; 95 \%$ CI: $-2.57,-0.10$; $P=0.0349$ ). For subjects with moderate to no sleep disturbance at baseline, the change in ESS total score was not significant at any time point. Placebo response was similar between subgroups. There was a trend for a larger decrease (improvement) in ESS total score in subjects receiving GEn with very severe to severe sleep disturbance at baseline compared with those with moderate to no sleep disturbance.

\section{Discussion}

This integrated analysis indicates that, compared with placebo, once-daily GEn $1200 \mathrm{mg}$ significantly improves subjective sleep outcomes in subjects with moderate to severe primary RLS suffering from varying degrees of 
Very severe to severe sleep disturbance: GEn $1200 \mathrm{mg}$

Moderate to no sleep disturbance: GEn $1200 \mathrm{mg}$

A

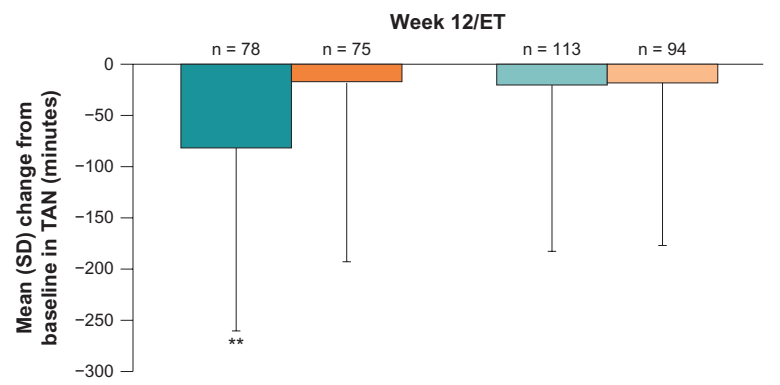

Very severe to severe sleep disturbance: $\mathrm{PBO}$

Moderate to no sleep disturbance: PBO

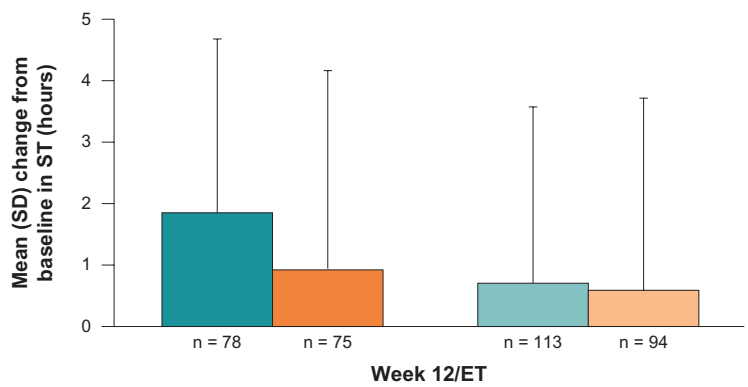

Figure 3 Change from baseline in novel sleep endpoints at Week I2/ET (modified intent-to-treat population): (A) mean (SD) change from baseline in TAN (minutes), OC and (B) mean change from baseline (SD) in ST (hours), OC.

Note: $* * P \leq 0.001$.

Abbreviations: ET, early termination; GEn, gabapentin enacarbil; OC, observed case; PBO, placebo; SD, standard deviation; ST, sleep time; TAN, time awake during the night.

sleep disturbance. This was demonstrated by results from the MOS Sleep Scale, with a significant improvement in all domains; the PSQ, with significant improvement in sleep quality (both the moderate to no sleep disturbance and the very severe to severe sleep disturbance groups), as well as nighttime awakenings and hours awake per night (very severe to severe sleep disturbance group only); and the PghSD, with significant improvement in WASO. The MOS Sleep Scale and PghSD results confirm those already reported in the individual studies; ${ }^{13,14}$ for the PSQ, the individual studies reported significant improvements for GEn $1200 \mathrm{mg}$ and $600 \mathrm{mg}$ compared with placebo on all items. ${ }^{13,14}$

It is unsurprising that subjects with very severe to severe sleep disturbance at baseline reported less ST and TST and longer TAN and WASO on the 24-Hour RLS Symptom Diary and PghSD at baseline compared with subjects with moderate to no sleep disturbance. However, with the PghSD, more analyses yielded a significant treatment difference in favor of GEn versus placebo, regardless of sleep disturbance at baseline compared with results from the 24-Hour RLS Symptom Diary. Moreover, there were differences in reported values between the two assessment tools. The PghSD allows for greater fidelity of estimated sleep endpoints compared with the 24-Hour RLS Symptom Diary because it uses open text fields to collect data (subjects indicated an exact number of minutes they were awake during the night), and more frequent collection intervals were used in these two studies. In contrast, the 24-Hour RLS Symptom Diary collected data
Very severe to severe sleep disturbance: GEn $1200 \mathrm{mg}$ Moderate to no sleep disturbance: GEn $1200 \mathrm{mg}$

A

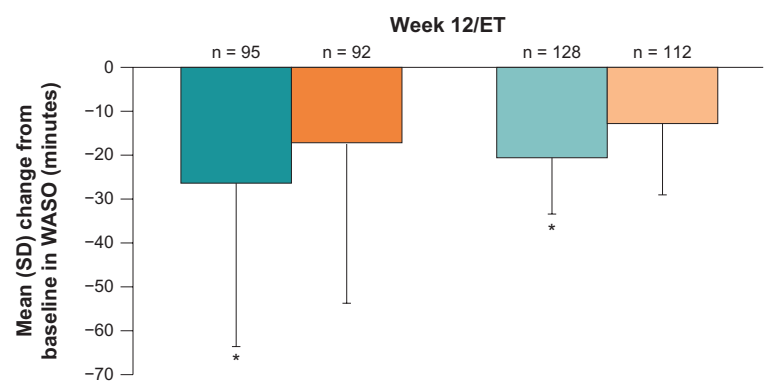

Very severe to severe sleep disturbance: PBO

Moderate to no sleep disturbance: PBO

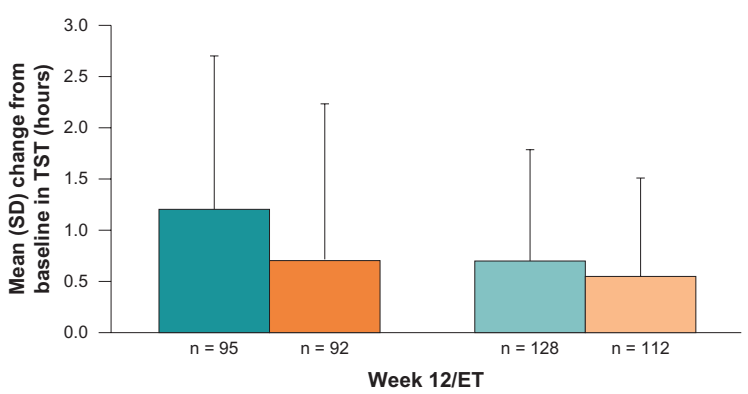

Figure 4 Change from baseline in Pittsburgh Sleep Diary sleep endpoints at Week I2/ET (modified intent-to-treat population): (A) mean (SD) change from baseline in WASO (minutes), LOCF and (B) mean (SD) change from baseline in TST (hours), LOCF.

Note: $* P \leq 0.01$.

Abbreviations: ET, early termination; GEn, gabapentin enacarbil; LOCF, last observation carried forward; PBO, placebo; SD, standard deviation; TST, total sleep time; WASO, wake time after sleep onset. 

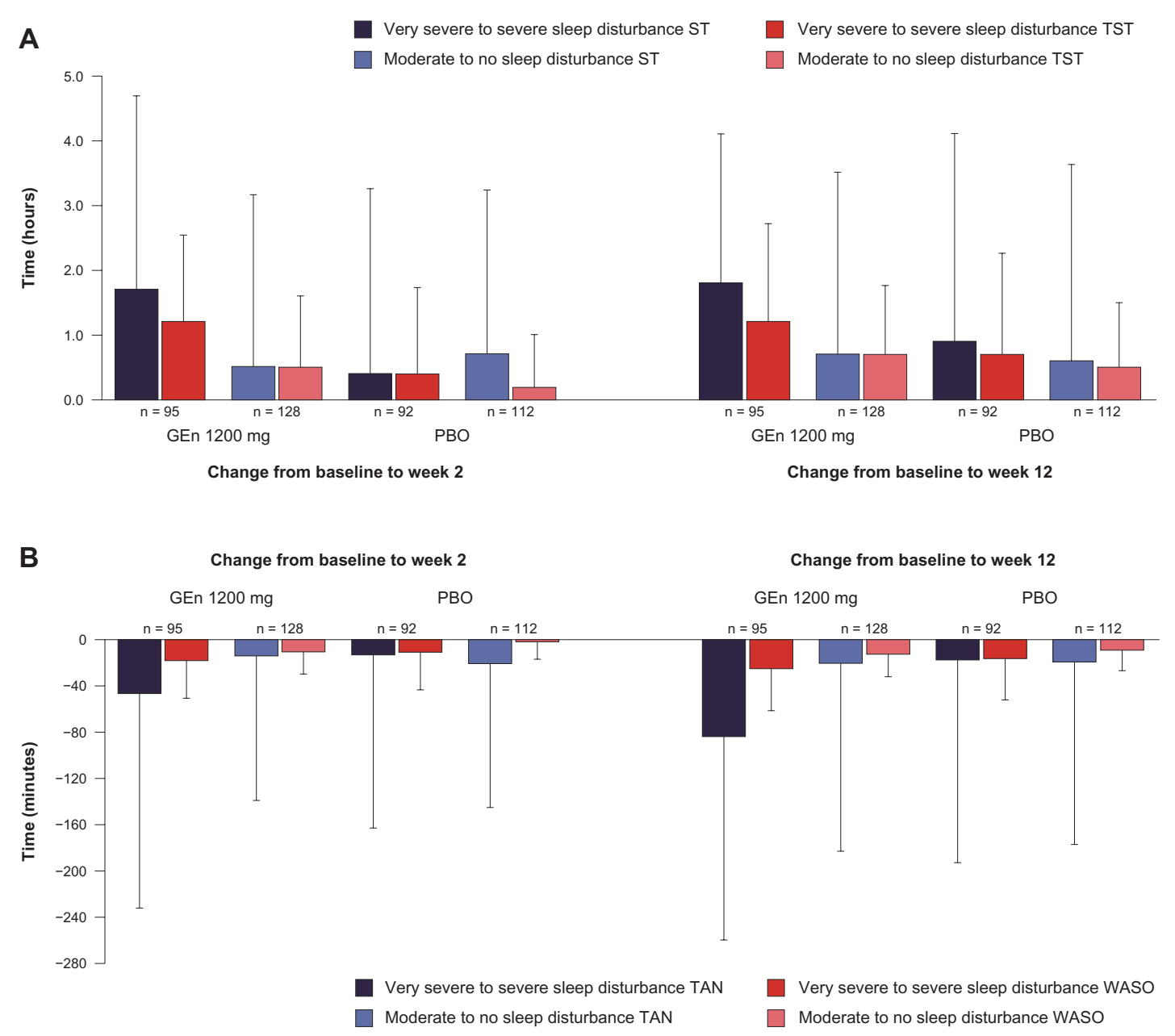

Figure 5 Comparison of novel sleep endpoints derived from the 24-Hour RLS Symptom Diary and the PghSD by sleep disturbance subgroup (modified intent-to-treat population): (A) mean (SD) change from baseline in ST (24-Hour RLS Symptom Diary) versus TST (PghSD) and (B) mean (SD) change from baseline in TAN (24-Hour RLS Symptom Diary) versus WASO (PghSD).

Abbreviations: GEn, gabapentin enacarbil; PBO, placebo; ST, sleep time; TAN, time awake during the night; TST, total sleep time; WASO, wake time after sleep onset.

in 30-minute intervals over a single 24-hour period prior to a visit (if a subject indicated they were awake at a particular interval, they were counted as being awake for the entire 30-minute period, even if in reality they were only awake for a portion of it). In addition, in the absence of a diary entry, it was assumed the subject was awake. This could have resulted in the subject's awake time being overestimated when using this tool. Although the PghSD is a well-established, validated tool for quantifying sleep behavior that has been shown to correlate well with other measures, ${ }^{18,19}$ it is generally agreed that patients underestimate ST, and diaries may be a more accurate measure. While the 24-Hour RLS Symptom Diary allows assessment of onset and severity of RLS symptoms, the sleep measure component, as currently constructed, offers no clear advantage over traditional measures. However, with modifications to allow higher resolution of ST and enhanced patient compliance, use of the diary may be more intuitive to patients because it reflects a continuum of RLS symptoms and ultimately translate into a clinical tool for patient management.

The number of subjects reporting somnolence and/or sedation AEs was similar in both baseline sleep disturbance subgroups, although there was a greater improvement in ESS total score in subjects with very severe to severe sleep disturbance than in those with moderate to no sleep disturbance. Few subjects withdrew due to somnolence/sedation AEs: one in the very severe to severe sleep disturbance group and three in the moderate to no sleep disturbance subgroup. No subjects in either subgroup of the placebo group withdrew because of somnolence/sedation.

\section{Limitations}

Although this analysis of integrated data allows an evaluation of sleep outcomes in a large group of subjects by degree of 
sleep disturbance at baseline, there are several limitations to the analysis and to the conclusions that may be drawn from it. First, this analysis used data only from subjects receiving GEn $1200 \mathrm{mg}$, although the daily dose of GEn approved by the US Food and Drug Administration for the treatment of moderate to severe primary RLS in adults is $600 \mathrm{mg}$. The $600 \mathrm{mg}$ dose was evaluated in Study XP053 but not in Study XP052, so it could not be included in the integrated analysis with pooled data from the two trials. Another limitation is that the PghSD was assessed at Weeks 2, 4, 8, and 12, whereas the 24-Hour RLS Symptom Diary was assessed only at Weeks 2 and 12; therefore, these two instruments could be compared only at Weeks 2 and 12. Further, the recall periods differ between the PghSD and the 24-Hour RLS Symptom Diary, preventing a direct comparison at a given time. Although the study population has been categorized into subjects with very severe to severe and moderate to no sleep disturbance, this classification is based on one item of the IRLS relating to the severity of sleep disturbance. Subgroups could have been created based on the response to other items of the IRLS or on other scales.

\section{Conclusion}

GEn significantly improves subjective sleep outcomes compared with placebo in subjects with moderate to severe primary RLS, regardless of the severity of sleep disturbance at baseline. A greater treatment benefit is seen in subjects with very severe to severe sleep disturbance than in those with moderate to no sleep disturbance on the IRLS total score. Although similar patterns were observed between treatment groups when comparing sleep endpoints derived from the PghSD and the 24-Hour RLS Symptom Diary, the PghSD reported more ST than the 24-Hour RLS Symptom Diary. It is probable that the PghSD allows for a more sensitive evaluation of sleep in subjects with moderate to severe primary RLS - to confirm this, both measures would need to be studied further, with correlation to an objective measure such as actigraphy or polysomnography.

\section{Acknowledgments}

The authors thank Sarah Brown BSc (Hons) and Joanna Wright DPhil (Caudex Medical, Oxford, UK), funded by GlaxoSmithKline, for assistance in preparing the initial draft of the manuscript, collating the comments of authors, and assembling tables and figures. The authors also wish to acknowledge the following individuals for critical review during the development of this manuscript: Susan VanMeter,
MD (GlaxoSmithKline [GSK]) for medical review and Gary Evoniuk, $\mathrm{PhD}$ (GSK).

\section{Disclosure}

Research funding for the design and conduct of these studies, and the collection, management, analysis, and interpretation of the data were sponsored by XenoPort. The preparation, review, and approval of the manuscript were sponsored by GSK and XenoPort.

$\mathrm{R}$ Bogan is a shareholder and employee of SleepMed and a consultant to Cephalon, GSK, Jazz, ApniCure, UCB, and Sunovion. He has participated in industry-funded research for Actelion, Boehringer Ingelheim, Cephalon, GSK, Jazz, Vanda, Merck, Pfizer, Schwartz, Sepracor, XenoPort, Ventus, Philips, ResMed, ApniCure, Sensory Medical, Johnson \& Johnson, and Apnex, and has participated in speaker bureaus for Cephalon, Jazz, Sunovion, and GSK. A Ellenbogen has received compensation from Allergan, GSK, Impax Pharmaceuticals, Novartis, and Teva Neuroscience. P Becker has received compensation from GSK, Pfizer, Sanofi-Aventis, Sepracor, and Schwartz. $\mathrm{C}$ Kushida has received financial support for research activities through research contracts between Stanford University and GSK, XenoPort, Merck, PacificMedico, and Ventus. E Ball has acted as a consultant to GSK and has participated in industry-funded research for GSK and Pfizer. W Ondo has participated in speaker bureaus for GSK, Allergan, Teva, Merz, Lundbeck, Avanir, and Ipsen. C Caivano and $\mathrm{S}$ Kavanagh are employees of GSK.

\section{References}

1. Allen RP, Picchietti D, Hening WA, Trenkwalder C, Walters AS, Montplaisi J; Restless Legs Syndrome Diagnosis and Epidemiology workshop at the National Institutes of Health; International Restless Legs Syndrome Study Group. Restless legs syndrome: diagnostic criteria, special considerations, and epidemiology. A report from the restless legs syndrome diagnosis and epidemiology workshop at the National Institutes of Health. Sleep Med. 2003;4(2):101-119.

2. Allen RP, Walters AS, Montplaisir J, et al. Restless legs syndrome prevalence and impact: REST general population study. Arch Intern Med. 2005;165(11):1286-1292.

3. Montplaisir J, Boucher S, Poirier G, Lavigne G, Lapierre O, Lespérance P. Clinical, polysomnographic, and genetic characteristics of restless legs syndrome: a study of 133 patients diagnosed with new standard criteria. Mov Disord. 1997;12(1):61-65.

4. Saletu B, Gruber G, Saletu M, et al. Sleep laboratory studies in restless legs syndrome patients as compared with normals and acute effects of ropinirole. 1. Findings on objective and subjective sleep and awakening quality. Neuropsychobiology. 2000;41(4):181-189.

5. Hening W, Walters AS, Allen RP, Montplaisir J, Myers A, Ferini-Strambi L. Impact, diagnosis and treatment of restless legs syndrome (RLS) in a primary care population: the REST (RLS epidemiology, symptoms, and treatment) primary care study. Sleep Med. 2004;5(3):237-246. 
6. Hornyak M, Feige B, Voderholzer U, Philipsen A, Riemann D. Polysomnography findings in patients with restless legs syndrome and in healthy controls: a comparative observational study. Sleep. 2007;30: 861-865.

7. Brand S, Lehtinen A, Hatzinger M, Holsboer-Trachsler E. Comparison of sleep EEG profiles of patients suffering from restless legs syndrome, restless legs syndrome and depressive symptoms, and major depressive disorders. Neuropsychobiology. 2010;61(1):41-48.

8. Brand S, Beck J, Hatzinger M, Savic M, Holsboer-Trachsler E. Unfavorable polysomnographic sleep patterns predict poor sleep and poor psychological functioning 3 years later in patients with restless legs syndrome. Neuropsychobiology. 2011;63(2):92-102.

9. Horizant ${ }^{\circledR}$ (gabapentin enacarbil) Extended-Release Tablets prescribing information. Research Triangle Park, NC: GlaxoSmithKline. Available from: http://us.gsk.com/products/assets/us_horizant.pdf. Accessed July 8, 2011.

10. Cundy KC, Annamalai T, Bu L, et al. XP13512 [(+/-)-1-([(alphaisobutanoyloxyethoxy)carbonyl] aminomethyl)-1-cyclohexane acetic acid], a novel gabapentin prodrug: II. Improved oral bioavailability, dose proportionality, and colonic absorption compared with gabapentin in rats and monkeys. J Pharmacol Exp Ther. 2004;311(1):324-333.

11. Cundy KC, Branch R, Chernov-Rogan T, et al. XP13512 [(+/-)-1-([(alpha-isobutanoyloxyethoxy)carbonyl] aminomethyl)-1cyclohexane acetic acid], a novel gabapentin prodrug: I. Design, synthesis, enzymatic conversion to gabapentin, and transport by intestinal solute transporters. J Pharmacol Exp Ther. 2004;311(1):315-323.

12. Cundy KC, Sastry S, Luo W, Zou J, Moors TL, Canafax DM. Clinical pharmacokinetics of XP13512, a novel transported prodrug of gabapentin. J Clin Pharmacol. 2008;48(12):1378-1388.

13. Kushida CA, Becker PM, Ellenbogen AL, Canafax DM, Barrett RW; XP052 Study Group. Randomized, double-blind, placebo-controlled study of XP13512/GSK1838262 in patients with RLS. Neurology. 2009;72(5):439-446.
14. Lee DO, Ziman RB, Perkins AT, Poceta JS, Walters AS, Barrett RW; XP053 Study Group. A randomized, double-blind, placebo-controlled study to assess the efficacy and tolerability of gabapentin enacarbil in subjects with restless legs syndrome. J Clin Sleep Med. 2011;7(3): 282-292.

15. GlaxoSmithKline. XP13512 (GSK1838262) versus placebo in patients with restless legs syndrome. In: ClinicalTrials.gov [website on the Internet]. Bethesda, MD: US National Library of Medicine; 2006 [updated May 10, 2011]. Available from: http://www.clinicaltrials.gov/ ct2/show/NCT00298623. NLM identifier: NCT00298623. Accessed March 29, 2013.

16. GlaxoSmithKline. XP13512 vs placebo in patients with restless legs syndrome. In: ClinicalTrials.gov [website on the Internet]. Bethesda, MD: US National Library of Medicine; 2006 [updated May 10, 2011]. Available from: http://www.clinicaltrials.gov/ct2/show/NCT00365352. NLM identifier: NCT00365352. Accessed March 29, 2013.

17. Walters AS, LeBrocq C, Dhar A, et al; International Restless Legs Syndrome Study Group. Validation of the International Restless Legs Syndrome Study Group rating scale for restless legs syndrome. Sleep Med. 2003;4(2):121-132.

18. Monk TH, Reynolds CF, Kupfer DJ, et al. The Pittsburgh Sleep Diary. J Sleep Res. 1994;3(2):111-120.

19. Monk TH, Buysse DJ, Kennedy KS, Pods JM, DeGrazia JM, Miewald JM. Measuring sleep habits without using a diary: the sleep timing questionnaire. Sleep. 2003;26(2):208-212.

20. Hays RD, Stewart AL. Sleep measures. In: Stewart AL, Ware JE, editors. Measuring Functioning and Well-Being: The Medical Outcomes Study Approach. Durham and London, UK: Duke University Press; 1992:235-259.
Journal of Parkinsonism \& Restless Legs Syndrome

\section{Publish your work in this journal}

Journal of Parkinsonism and Restless Legs Syndrome is an online, open access, peer-reviewed journal. The journal publishes review articles, historical reviews, original research articles, case reports, letters to the editor, clinical teaching cases, neuroradiology highlights, neuropathology highlights, neuropsychiatry highlights, autobiographies, conference

\section{Dovepress}

proceedings, abstracts and book reviews. The manuscript management system is completely online and includes a very quick and fair peerreview system, which is all easy to use. Visit http://www.dovepress.com testimonials.php to read real quotes from published authors. 